\title{
D. Department of
}

Journal of Rehabilitation Research and Development

Vol. 40, No. 5, September/October 2003

Pages 443-454

\section{Clinical evaluation of Functional Electrical Therapy in acute hemiplegic subjects}

\author{
Mirjana B. Popovic, PhD; Dejan B. Popovic, PhD, DrTech; Thomas Sinkjær, PhD, DrMed; \\ Aleksandra Stefanovic, MD; Laszlo Schwirtlich, PhD, MD \\ Center for Sensory-Motor Interaction (SMI), Aalborg University, Denmark; Institute for Rehabilitation "Dr. Miroslav \\ Zotovic,” Belgrade, Yugoslavia; Center for Multidisciplinary Studies, University of Belgrade, Belgrade, Yugoslavia
}

\begin{abstract}
This paper describes a clinical randomized singleblinded study of the effects of Functional Electrical Therapy (FET) on the paretic arms of subjects with acute hemiplegia caused by strokes. FET is an exercise program that comprises voluntary arm movements and opening, closing, holding, and releasing of objects that are assisted by a neural prosthesis (electrical stimulation). FET consisted of a 30 min everyday exercise for 3 consecutive weeks in addition to conventional therapy. Twenty-eight acute hemiplegic subjects participated in a 6 mo study. The subjects were divided into lower functioning groups (LFGs) and higher functioning groups (HFGs) based on their capacity to voluntarily extend the wrist and fingers against the gravity, and were randomly assigned to controls or FET groups. The outcomes included the Upper Extremity Function Test, the coordination of elbow and shoulder movements, spasticity of key muscles of the paretic arm, and Reduced Upper Extremity Motor Activity Log. FET and control groups showed a recovery trend in all outcome measures. The gains in FET groups were much larger compared with the gains in control groups. The speed of recovery in FET groups was substantially faster compared with the recovery rate in control groups during the first 3 weeks (treatment). The LFG subjects showed less improvement than the HFG in both the FET and control groups.
\end{abstract}

Key words: acute hemiplegia, electrical stimulation, exercise, paretic arm and hand, therapy.

\section{INTRODUCTION}

Hemiplegic subjects are often unable to functionally use one arm and hand. This disability leads to the development of compensatory strategies for accomplishing daily needs and frequently minimizes the use of the paretic arm and hand. The clinical evaluations of electrical stimulation-based rehabilitation strongly suggest that electrical stimulation promotes functional recovery of reaching and grasping [1-4]. Many new rehabilitation treatments were designed to enhance the concentrated use of the paretic arm and hand (e.g., intensive exercise [5,6], Constrained Induced Movement Therapy (CIMT) [7-9], robot-induced therapy [10], and electrical stimulation [11-18]).

\footnotetext{
Abbreviations: ANOVA = analysis of variance, CIMT $=$ Constrained Induced Movement Therapy, CNS = central nervous system, $\mathrm{CT}=$ computer tomography, $\mathrm{CVA}=$ cerebrovascular accident, DT $=$ Drawing Test, FET $=$ Functional Electrical Therapy, HFG = higher functioning group, LFG = lower functioning group, $\mathrm{MRI}=$ magnetic resonance imaging, $\mathrm{RUE} / \mathrm{MAL}=$ Reduced Upper Extremity Motor Activity Log, SD = standard deviation, UEFT = Upper Extremity Functioning Test.

This material was based on work supported partly by the Danish National Research Foundation, Copenhagen, Denmark, and the Ministry for Science and Technology of Serbia, Belgrade, Serbia.

Address all correspondence and requests for reprints to Professor Mirjana B. Popovic, Center for Sensory-Motor Interaction (SMI), Aalborg University, Fredrik Bajers Vej 7D-3, 9220 Aalborg, Denmark; +45-9-635-8758, fax: +45-9-815-4008; email: mpo@ smi.auc.dk.
} 
Journal of Rehabilitation Research and Development Vol. 40, No. 5, 2003

Functional Electrical Therapy (FET) combines intensive voluntary activation of proximal muscles and patterned multichannel electrical stimulation of distal muscles providing grasp and release functions in the paretic hand [19-22]. The essential difference between FET and other electrical stimulation methods is that while electrical stimulation assists the opening, closing, and releasing functions, in parallel, a hemiplegic subject can concentrate on manipulation, that is, on shoulder and elbow movements. This added ability to grasp and release objects motivates a hemiplegic subject to exercise in a functional manner, i.e., to practice typical movements that were part of his or her normal daily activities before the cerebrovascular accident (CVA). In addition, the electrical stimulation in FET sends strong signals to the central nervous system (CNS) that are superimposed to the proprioceptive and exteroceptive sensory activity that occurs during the functional tasks.

The hypothesis in this study was that the FET results in substantial carryover effects if applied in acute hemiplegic subjects. This hypothesis was based on two elements: (1) the CNS is slowly changing its structure and organization; thus, the externally added exercise promotes functional recovery and (2) the electrically induced grasping, holding, and releasing of objects allows acute hemiplegics to perform functions, thereby, motivating them to use the paretic arm and reduce the development of compensatory mechanisms typical for the post-CVA period. In this paper, we show results from a randomized singleblinded study of FET in 28 acute hemiplegic subjects.

\section{METHODS AND MATERIALS}

Forty-one acute inpatient hemiplegic subjects were considered for the study (in average $7 \pm 2$ weeks after the CVA with $59.9 \pm 9.3$ mean age \pm SD [standard deviation]). Twenty-eight of these subjects were randomly assigned to a control or FET group. All study subjects received the same amount of conventional daily therapy during the treatment and follow-up, that is, in total during 26 weeks. In addition, during the first 3 weeks after entering into the study, all subjects participated in $30 \mathrm{~min}$ long treatment sessions. The $30 \mathrm{~min}$ sessions were either exercise with stimulation (FET group) or only exercise (control group). The subjects were assessed at the start of the study; after the treatment that lasted for 3 weeks; and at 6,13 , and 26 weeks of the follow-up.

\section{Subjects}

The subjects were accepted in the study in the acute phase after the CVA. The inclusion criteria for subjects included (1) having more than 2 weeks and less than 6 months following the first CVA that was caused by ischemia or hemorrhagia and confirmed by magnetic resonance imaging (MRI) or computer tomography (CT), (2) being above age 18, (3) being able to give informed consent, and (4) being able to understand how to apply electrical stimulation for controlling the grasp. The exclusion criteria were (1) dependent on care, prior to stroke, for activities of daily living; (2) severe medical condition in any arm and/or hand that precludes participation in the study; (3) previous injury or disease or contracture affecting paretic or nonparetic arm or hand; and (4) electrical life support devices (e.g., cardiac pacemaker).

These 41 hemiplegic subjects were characterized to either the lower functioning group (LFG) or higher functioning group (HFG). This division was used following the studies of CIMT [4,7]. The HFG hemiplegic subjects could actively extend the paretic wrist more than $20^{\circ}$ and extend their metacarpophalangeal (MP) and interphalangeal (IP) joints of all digits more than $20^{\circ}$. No upperfunctioning limit for the HFG was imposed. The LFG hemiplegic subjects could extend the paretic wrist between $10^{\circ}$ and $20^{\circ}$, and extend the MP and IP joints of the thumb and minimum of two other digits between $10^{\circ}$ and $20^{\circ}$. Seventeen subjects belonged to the LFG and twenty-four to the HFG. A random generator was used to select 28 subjects for the study: 16 hemiplegic subjects from HFG $(60.6 \pm 5.8$ years $)$ and 12 hemiplegic subjects from LFG (58.6 \pm 12.4 years). A random generator was again used to assign patients to the FET or control groups. As a result, hemiplegic subjects were assigned into four groups: (1) FET HFG, (2) control HFG, (3) FET LFG, and (4) control LFG (Table 1).

The study was blinded, since the evaluators did not know to which group (HFG or LFG, FET or control) subjects belonged. The hemiplegic subjects who were assigned to FET groups knew that they were electrically stimulated, since the stimulation resulted with fingers and thumb movements. Control group hemiplegic subjects were offered FET after the 26 weeks long study if they were interested. The local ethics committees approved the experimental procedure for this study in conformity with the Declaration of Helsinki. 
POPOVIC et al. Clinical evaluation of Functional Electrical Therapy

Table 1.

Basic data for hemiplegic subjects included in clinical randomized single-blinded study assessing efficacy of Functional Electrical Therapy (FET). Third column shows time between onset of stroke and admission to study in weeks. A random generator was used to form FET and control groups.

\begin{tabular}{|c|c|c|c|c|c|c|}
\hline Patient & Age (yr) & $\begin{array}{l}\text { Period Between Stroke and } \\
\text { Beginning of Study (wk) }\end{array}$ & Ashworth Grade & Paretic Side & CVA Type & Group \\
\hline 1 & 62 & 4 & $1+$ & Left & Ischemia & FET HGT \\
\hline 2 & 61 & 6 & $1+$ & Left & Hemorrhage & FET HGT \\
\hline 3 & 50 & 7 & $2-3$ & Left & Ischemia & FET HGT \\
\hline 4 & 52 & 11 & 4 & Right & Ischemia & FET HGT \\
\hline 5 & 65 & 8 & $2-3$ & Right & Ischemia & FET HGT \\
\hline 6 & 68 & 9 & $1+$ & Right & Ischemia & FET HGT \\
\hline 7 & 62 & 6 & 4 & Right & Ischemia & FET HGT \\
\hline 8 & 61 & 7 & 3 & Right & Ischemia & FET HGT \\
\hline 9 & 62 & 8 & $1+$ & Right & Hemorrhage & Control HFG \\
\hline 10 & 65 & 4 & 3 & Right & Ischemia & Control HFG \\
\hline 11 & 61 & 8 & 3 & Right & Ischemia & Control HFG \\
\hline 12 & 72 & 5 & $2-3$ & Left & Ischemia & Control HFG \\
\hline 13 & 61 & 5 & $2-3$ & Right & Ischemia & Control HFG \\
\hline 14 & 61 & 8 & 4 & Right & Hemorrhage & Control HFG \\
\hline 15 & 53 & 6 & $1+$ & Right & Ischemia & Control HFG \\
\hline 16 & 58 & 8 & 4 & Right & Ischemia & Control HFG \\
\hline 17 & 41 & 4 & $2-3$ & Right & Ischemia & FET LFG \\
\hline 18 & 37 & 5 & $2-3$ & Right & Ischemia & FET LFG \\
\hline 19 & 72 & 4 & 3 & Right & Hemorrhage & FET LFG \\
\hline 20 & 65 & 11 & 4 & Left & Ischemia & FET LFG \\
\hline 21 & 61 & 6 & 3 & Right & Ischemia & FET LFG \\
\hline 22 & 75 & 7 & $1+$ & Right & Ischemia & FET LFG \\
\hline 23 & 72 & 5 & 4 & Left & Ischemia & Control LFG \\
\hline 24 & 45 & 6 & $2-3$ & Right & Ischemia & Control LFG \\
\hline 25 & 54 & 11 & $1+$ & Right & Ischemia & Control LFG \\
\hline 26 & 63 & 5 & 4 & Right & Hemorrhage & Control LFG \\
\hline 27 & 51 & 5 & 2 & Right & Ischemia & Control LFG \\
\hline 28 & 67 & 6 & $2-3$ & Left & Ischemia & Control LFG \\
\hline
\end{tabular}

\section{Rehabilitation Treatments}

\section{FET Groups}

The subjects from the FET groups, in addition to conventional therapy, performed $30 \mathrm{~min}$ long exercise with the paretic arm and hand every day during 3 consecutive weeks. The conventional therapy was a comprehensive treatment introduced by Bobath [23]. This exercise was assisted with a neural prosthesis that controlled the opening, grasping, and releasing functions by mimicking natural movement. Four channels of electrical stimulation were applied via self-adhesive surface electrodes positioned over the following muscle groups: finger flexors $(m$. flexor digitorum profundus and $m$. flexor digitorum superficialis), finger extensors (m. extensor digitorum communis), thumb extensor ( $m$. extensor longus pollicis), and the thenar 
muscle group ( $m$. pollicis abductor and $m$. opponens). The positions of electrodes were carefully determined to maximize selectivity of stimulation by the experienced physical therapist. The typical stimulation parameters were frequency $50 \mathrm{~Hz}$, pulse duration $T=200 \mu \mathrm{s}$, and stimulation intensity $I=20 \mathrm{~mA}-45 \mathrm{~mA}$. The timing and intensity of stimulation were programmed to mimic as closely as possible the normal prehension, grasp, and release typical for a normal hand.

During FET sessions, the hemiplegic subjects were instructed to try to functionally use a toothbrush, comb, telephone receiver, pen, small food, $0.33 \mathrm{~L}$ can, $0.33 \mathrm{~L}$ bottle, $1 \mathrm{~L}$ container, $\mathrm{CD}$ (compact disc) for computer, and $0.25 \mathrm{~L}$ coffee mug; yet, they were not limited to these objects. The objects were selected so the subjects would be forced to practice palmar, lateral, and precision grasps. Functional use consisted of reaching, grasping, manipulating, and using an object; returning the object to the original post; and releasing the object. The hemiplegic subjects were instructed to trigger the opening synergy with their nonparetic hand at the appropriate time during the reaching phase and to trigger the release function once they accomplished effectively the task or established that they were not able to fulfill the task. During the 3 weeks of FET, the hemiplegic subjects started with easier tasks and included more difficult tasks upon their increased abilities. In most cases, the simplest tasks were the use of the can, telephone receiver, and similar round, middle-sized objects. The overall goal during a single session was to perform as many tasks as possible with the paretic arm.

A trained physical therapist assisted the subjects while they were trying to reach, grasp, and functionally use objects with their paretic hand and arm. The assistance comprised ensuring the hemiplegic subjects held the object in the adequate orientation and position, if required, and even more important, instructing them as to when and how to maximize the use of the externally controlled hand. FET sessions were sometimes performed without supervision after a subject learned how to position the stimulation electrodes correctly and how to appropriately train his or her paretic arm and hand.

FET sessions were performed 7 days a week within the rehabilitation institution since the study subjects were inpatients. The subjects occasionally missed FET sessions but never more then 2 days in a row.

\section{Control Groups}

The subjects from the control LFG and HFG received conventional physiotherapy. Thirty minutes of supervised daily exercise with the paretic arm and hand were added that included the same tasks as the FET groups, yet without a neural prosthesis.

\section{Outcomes Measures}

The outcome measures used to evaluate the effects of FET versus no FET were the (1) Upper Extremity Functioning Test (UEFT) [24,25], (2) Drawing Test (DT) [26,27], (3) modified Ashworth muscle spasticity scale of the key muscles of the paretic arm [28], and (4) Reduced Upper Extremity Motor Activity Log (RUE/MAL) questionnaire [29]. The UEFT was selected because it explicitly shows the subject's ability to perform typical daily activities. The DT was used because it directly shows the changes in the organization of control of proximal, nonstimulated sensory-motor mechanisms and implicitly shows the change of spasticity of the paretic arm. The RUE/MAL was applied to assess the users satisfaction. The same trained individual assessed each outcome to exclude intervariability.

The subjects were assessed at the start of the study; after the treatment that lasted for 3 weeks; and at 6,13, and 26 weeks after the start of treatment. The Ashworth scale grading and RUE/MAL were performed at the start and end of the study ( 0 and 26 weeks).

\section{Upper Extremity Function Test}

The UEFT determined differences in the performance of particular activities of daily living without a neural prosthesis. The following tasks were tested:

1. Combing hair.

2. Using a fork.

3. Picking up a VHS (Video Home System) format videotape.

4. Picking up a full juice can $(V=0.33 \mathrm{~L})$.

5. Picking up a full bottle $(V=0.33 \mathrm{~L})$.

6. Writing with a pen.

7. Using the telephone receiver.

8. Brushing teeth.

9. Pouring from a $1 \mathrm{~L}$ juice box.

10. Drinking from a mug $(V=0.25 \mathrm{~L})$.

11. Handling finger food. 
The selected activities include palmar grasp (tasks 4, 5, 7, and 9), lateral grasp (tasks 1, 2, 3, 8, and 10), and precision grip (tasks 6 and 11). The test evaluated the ability to handle small objects (tasks 1, 2, 5, 6, 8, 10, and 11), large objects (tasks 3, 4, 7, and 9), light objects (tasks 1, 2, 3, 6, 8, and 11), and heavy objects (tasks 4, 5, 7, 9, and 10).

The UEFT score was the number of successful repetitions of a task that a subject could perform during a 2 min period. A successful operation was one in which the subject grasped, manipulated, and used the object with his or her paretic arm. The same trained individual scored the UEFT by analyzing the video recordings from a session when the UEFT was performed. This action excluded intervariability from the evaluation. We also determined the averaged time to complete a task by dividing the 2 min interval with the number of successful repetitions.

\section{Drawing Test}

The DT shows the subject's ability to coordinate shoulder and elbow movements when the hand is moving in the horizontal plane within the typical workspace. The test relates to the coordination of muscles that have not been stimulated, yet have been activated voluntarily during the exercise.

The hemiplegic subjects were required to track a square $(20 \mathrm{~cm} \times 20 \mathrm{~cm})$ shown on the digitizing board with their paretic hand. The subjects were instructed not to move the trunk and shoulder (protraction-retraction) during the drawing. The movements were self-paced in clockwise and counterclockwise directions. The subjects were allowed to practice drawing on the digitizing board before assessment sessions.

The outcome measure was the ratio between the surface area surrounded by the drawn line and the surface of the square $\left(A=400 \mathrm{~cm}^{2}\right)$ expressed in percent. The higher score corresponded to better coordination between the paretic arm joints. We also calculated the time needed to draw the square. We determined the results presented in this paper by averaging recordings from the three consecutive trials. The DT was first used in a study that included subjects with no known neurological deficit and then in a pilot study with hemiplegic subjects $[26,27]$.

\section{Ashworth Muscle Spasticity Scale}

The Ashworth spasticity scale was applied to express the tonus of key muscles of the paretic arm. The same experienced physical therapist evaluated the Ashworth grades.

\section{Reduced Upper Extremity Motor Activity Log}

This test was a structured interview that examines how much and how well the subjects use their paretic arm outside of the laboratory setting. The subjects were rated on the amount they used their paretic arm ("Amount" Scale) and the quality of their movement during the functional activities ("How Well" Scale). The best scores in "Amount" and "How Well" scales were 60, with the worst at 0 . The subjects were instructed that the questions were about what they actually did outside of the laboratory setting, not what they think they were able to do with their paretic arm. The RUE/MAL questionnaire included the following 12 activities:

1. Pick up phone.

2. Open a door.

3. Eat finger foods.

4. Control the bathroom faucet.

5. Pick up a glass, bottle, or can.

6. Brush teeth.

7. Use a key to unlock the door.

8. Write on a paper.

9. Use the removable computer storage media (CD or floppy disk).

10. Use utensils for eating.

11. Pick up a cup by the handle.

12. Carry an object in the hand.

\section{Statistical Design and Sample Size Calculations}

We performed the Mann-Whitney rank sum test to compare the age, period between the CVA and start of treatment, and the initial functioning (the UEFT) of the hemiplegic subjects at the entry point to the study. For each of the primary endpoint variables (UEFT scores, DT scores, Ashworth grades, and RUE/MAL scores), we conducted the one-way analysis of variance (ANOVA). The within-subject factor was the time (start of study: baseline; after the treatment: 3 weeks; and 6, 12, and 26 weeks after the baseline). The primary test of the treatment was a test of the difference between the groups at 26 weeks after the start of the study.

\section{RESULTS}

The Mann-Whitney rank sum test showed that the age $\left(p_{\mathrm{HFG}}=0.613, p_{\mathrm{LFG}}=0.983\right)$, period between the onset of 
stroke and start of the treatment $\left(p_{\mathrm{HFG}}=0.447, p_{\mathrm{LFG}}=\right.$ $0.818)$, and the initial values of the UEFT $\left(p_{\mathrm{HFG}}=0.721\right.$, $\left.p_{\mathrm{LFG}}=0.1 .00\right)$ in the FET and control groups were similar. The UEFT for FET and control groups for HFG is shown in Figure 1(a) and for LFG in Figure 1(b). The averaged number of successful repetitions (mean $\pm \mathrm{SD}$ ) for the HFG at the start of the study was similar: $5.8 \pm 4.3$ (FET) and $4.9 \pm 1.3$ (controls); after the treatment, a substantial difference was found: $18.8 \pm 10.9$ (FET) and $9.6 \pm 6.3$ (controls); and at the end of 26 weeks, the UEFT score reached: $29.9 \pm 9.7$ (FET) and $15.4 \pm 7.6$ (controls). For the LFG, the averaged number of successful repetitions was at start 0 for both the FET and controls; after the treatment, $1.9 \pm 1.1$ (FET) and $0.2 \pm 0.1$ (controls); and at the end of 26 weeks, it reached $4.9 \pm 3.1$ in the FET group,

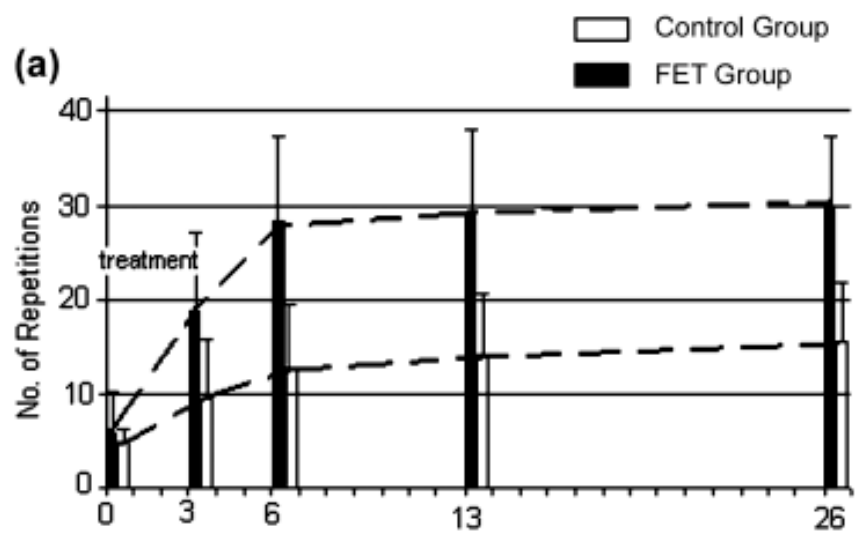

(b)

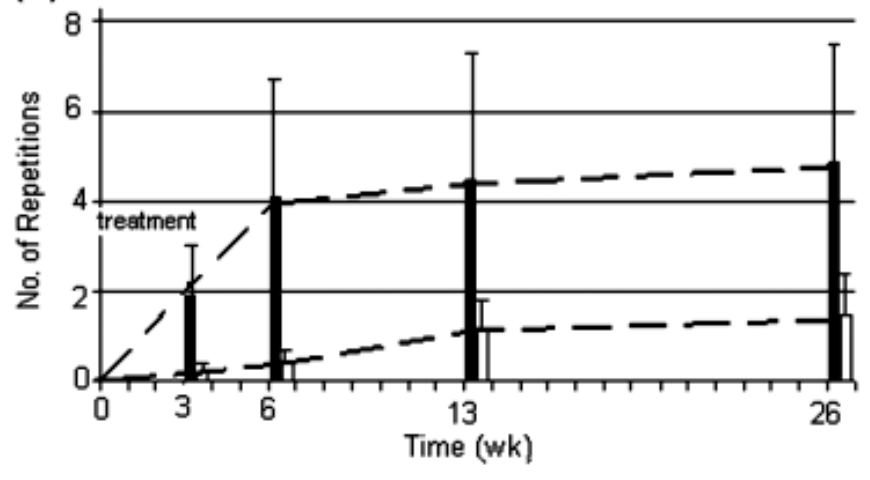

Figure 1.

Upper Extremity Function Test. Bars show averaged numbers of successful repetitions that acute hemiplegic subjects accomplished during a 2 min period with their paretic arm and hand. Averaging was done over all 11 tasks and in all subjects from LFG and HFG at start of treatment, and after 3, 6, 13, and 26 weeks. (a) Results in HFG and (b) results in LFG are shown. Same subjects performed about 30 repetitions with nonparetic hand and arm during a 2 min interval. Lines on top of bars are SDs. Trend lines are presented as dashed lines. and $1.5 \pm 0.9$ in the control group. Trend lines depicted as dashed lines in Figure $\mathbf{1}$ show the tendency of the improvement for all groups. Table 2 comprises the oneway ANOVA of the UEFT results between the FET and control groups.

Figure 2 shows the drawing ability of subjects from controls ((a) and (c)) and FET ((b) and (d)). These drawings include the recordings at the start of the study ( 0 weeks), after treatment ( 3 weeks), and at the end of the study (26 weeks). Visual inspection shows that all subjects improved. Subjects 23 and 17 from the LFG (Figure 2(c) and (d)) were not able to draw a figure that resembled a square at start, but after 3 weeks of treatment and even more so after 26 weeks, drawn patterns improved toward the goal. Subjects 11 and 3 from the HFG (Figure 2(a) and (b)) were able to draw a rectangle that converged to the target square during the course of clinical trial. Substantially higher improvement in subjects 3 and 17 was after FET compared with subjects 11 and 23 who were assigned to controls.

Figure 3 shows the DT results at start of the study and after 3, 6, 13, and 26 weeks for (a) HFG and (b) LFG FET and controls. The hemiplegic subjects from LFG were able to cover only a small fraction of the desired squared area (FET group: $16.2 \% \pm 9.5 \%$; control group: $14.2 \% \pm 6.8 \%$ ). After 26 weeks, the subjects from both groups improved in average their elbow versus shoulder coordination that resulted in much larger drawn areas (FET group: $49.8 \% \pm 9.6 \%$, control group: $36.7 \% \pm 6.9 \%$ ). The DT in the HFG groups showed the following trend: the FET group started with $43.9 \pm 10.3$ percent and reached $83.8 \pm 5.6$ percent at the end of clinical trial, while the control group started with $42.8 \pm 7.9$ percent and ended with $68.7 \pm 11.7$ percent of a square. The total improvements across the 26 weeks were $39.9 \pm 8.1$ percent in FET group, and $25.9 \pm 8.6$ percent in control group. Trend lines depicted as dashed lines in Figure $\mathbf{3}$ show the tendency of the improvement for all four groups. Table 3 comprises the DT results of a one-way ANOVA between the FET and control groups.

Movement in average became faster after the 3 weeks of treatment and improved even more across the 26 weeks. The LFG subjects could in average track the square in $9.1 \mathrm{~s} \pm 8.3 \mathrm{~s}$ (FET group) and in $11.6 \mathrm{~s} \pm 8.6 \mathrm{~s}$ (control group) at the end of the study (26 weeks) compared with $18.4 \mathrm{~s} \pm 9.5 \mathrm{~s}$ (FET group) and $17.9 \mathrm{~s} \pm 11.7 \mathrm{~s}$ (control group) at start of the study. The hemiplegic 
Table 2.

Averaged number of successful repetitions (using ANOVA of Upper Extremity Function Test) that FET and control groups were able to accomplish during 2 min intervals. Averaging was performed over all 11 tasks for all subjects from each group at start and after 3, 6, 13, and 26 wks of study.

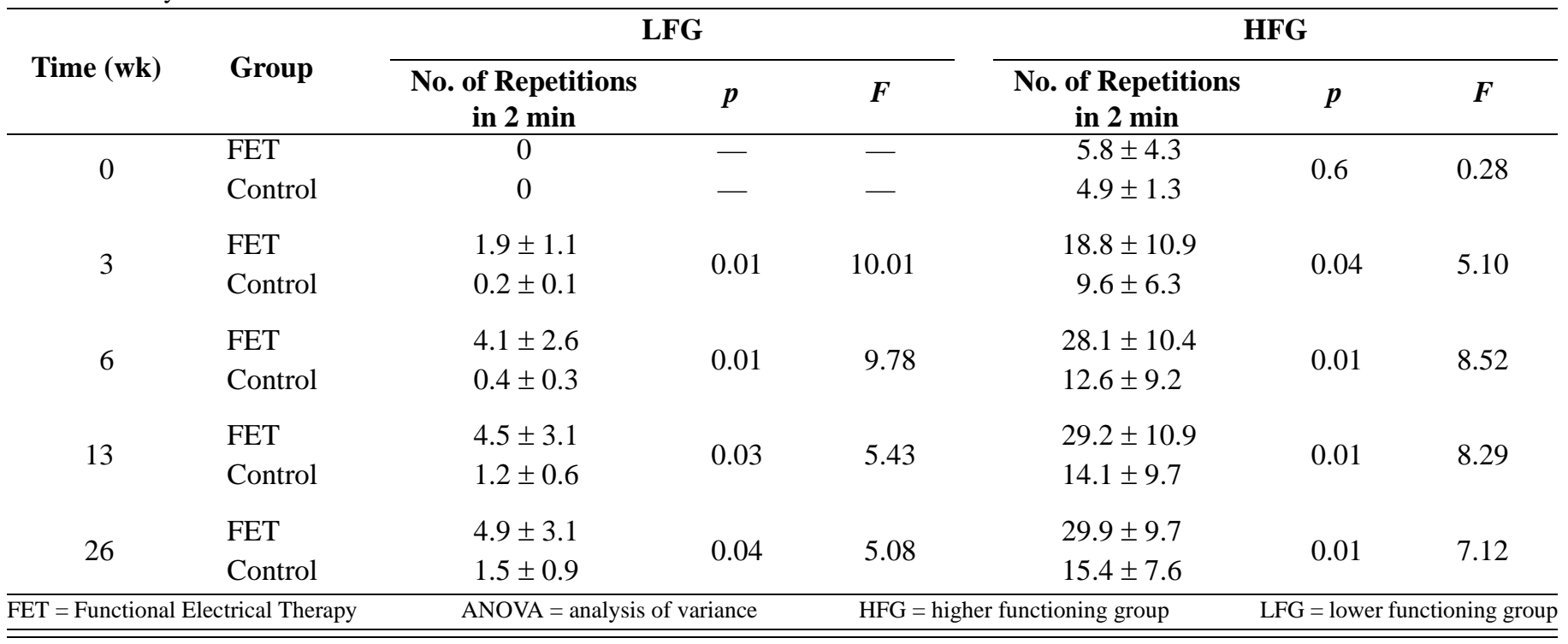
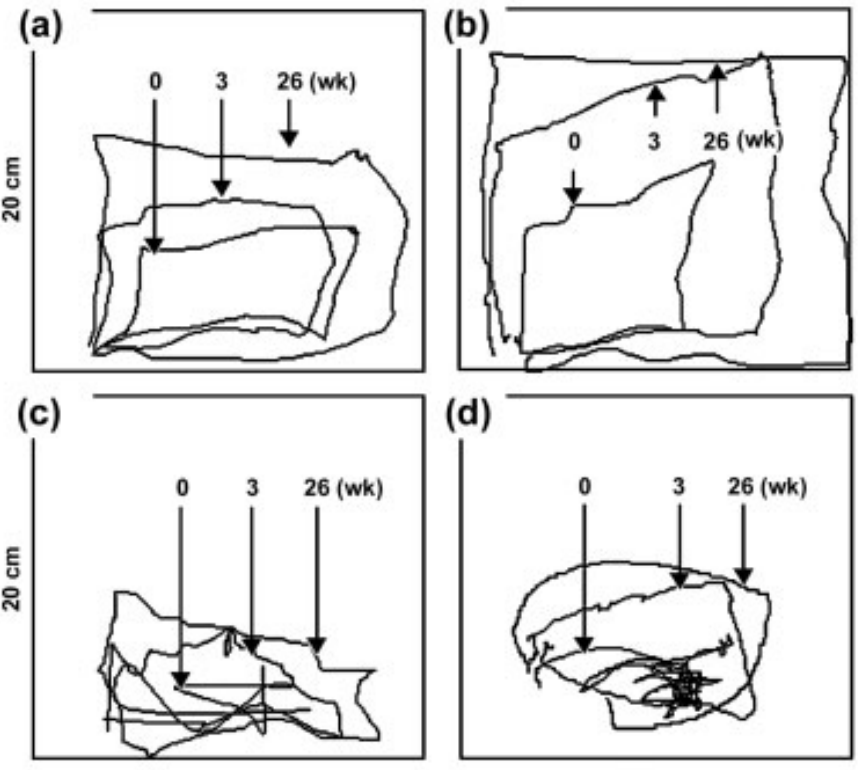

$20 \mathrm{~cm}$

(d)

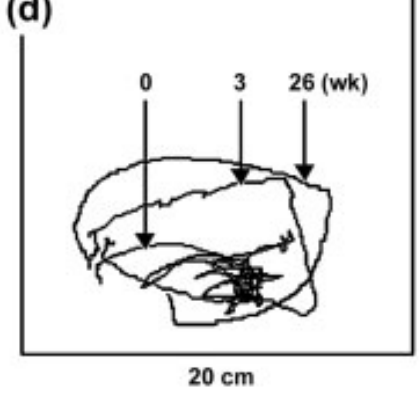

Figure 2.

Examples of drawing abilities of four subjects belonging to four different groups: (a) Subject 11, control HFG; (b) subject 3, FET HFG; (c) subject 23, control LFG; and (d) subject 17, FET LFG. Task was to track a square $(20 \mathrm{~cm} \times 20 \mathrm{~cm})$. Drawings shown were recorded at start ( 0 weeks) and after 3 and 26 weeks. All subjects improved their drawing abilities. Improvements were much more expressed in subjects assigned to FET, suggesting that combined exercise and electrical stimulation during period of major spontaneous recovery highly promote recovery. subjects from the FET HFG could track the square in $4.1 \mathrm{~s} \pm 2.7 \mathrm{~s}$ at the end of the study (26 weeks) compared with $11.2 \mathrm{~s} \pm 6.8 \mathrm{~s}$ at start. The subjects from control HFG in average could track the square in $7.3 \mathrm{~s} \pm 3.6 \mathrm{~s}$ at the end of the study (26 weeks) compared with $11.8 \mathrm{~s} \pm$ $7.9 \mathrm{~s}$ at start. No differences were found in the velocity profiles when moving clockwise versus counterclockwise in all groups.

The assessment of spasticity using the Ashworth scale was performed at the start and at the end of the study only, i.e., at 0 and 26 weeks (Table 4). The muscle tonus was decreased in all subjects, yet the statistically significant change was found only in the subjects from the HFG who were subjected to FET. When comparing the FET versus control groups, we found a statistically significant difference ( $p<0.05, F=6.3$ ).

The users' satisfaction and appreciation were assessed by means of the RUE/MAL questionnaire. Table 5 summarizes the "Amount" Scale and "How well" Scale of the RUE/MAL. The hemiplegic subjects assigned to HFG rated their improvement from about 13 percent (both groups) of effective use of paretic arm and hand at the start of the study to about 60 percent (FET group) and 30 percent (control group) at the end of the study (26 weeks). A statistically significant difference was found among the groups ( $p<0.02, F=8.80$ ). The 


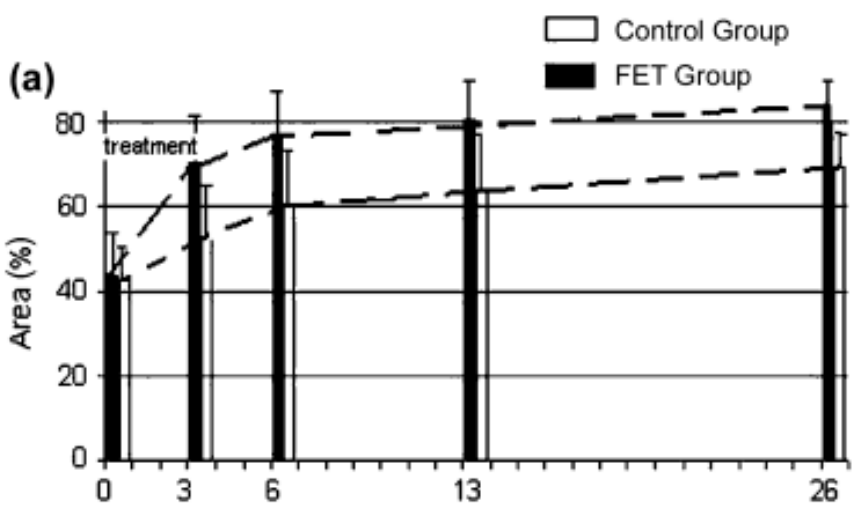

(b)

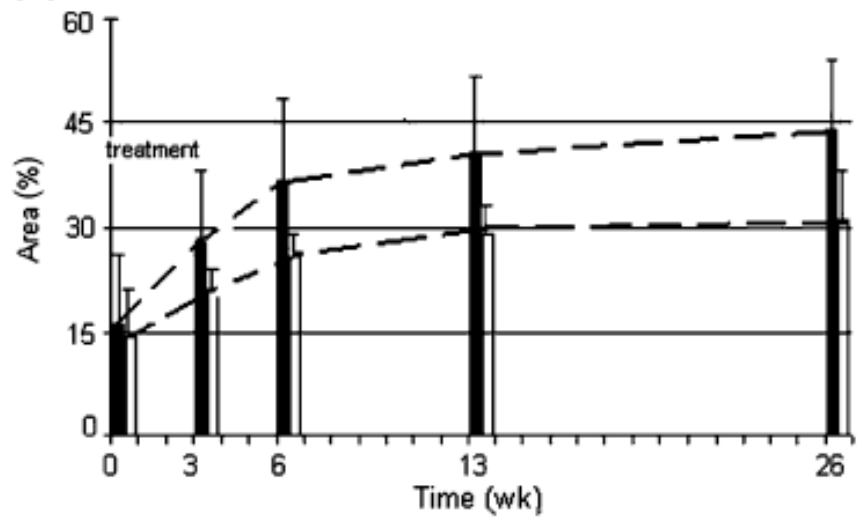

Figure 3.

Drawing Test. Bars show averaged normalized areas that hemiplegic subjects covered in DT with their paretic arm and hand at start and at 3, 6,13 , and 26 weeks. Averaging was done across all subjects from FET and control groups. Patients were asked to draw a square with their paretic arm and hand. Scores were obtained by dividing area of target square that patient drew with area of actual target square $(20 \times 20 \mathrm{~cm}=$ $400 \mathrm{~cm}^{2}$ ). Normalized area was calculated from coordinates recorded on digitizing board during DT. (a) Results in HFG and (b) results in LFG are shown. Larger area means that better coordination between shoulder and elbow joint was achieved. When same subjects drew with their nonparetic hand, they scored more than $90 \%$. Lines at top of bars show SDs. Trend lines are presented as dashed lines.

60 percent improvement is satisfactory when their side dominance (handedness) is considered. The hemiplegic subjects assigned to LFG started with zero functioning. They rated their improvement to 17 percent (FET group) compared with only about 3 percent (control group). The improvement of 17 percent in the FET LFG was still not good enough to make the paretic arm and hand functional; it was very likely that this arm and hand would not be used for daily activities.

\section{DISCUSSION AND CONCLUSIONS}

The FET is a novel treatment being different than earlier methods of electrical stimulation. The FET combines multichannel electrical stimulation that generates opening, grasping, and releasing functions with the exercise of the proximal muscles of the paretic arm. Stimulation pattern integrated in FET is timed to mimic the sequence of activation of muscles typical for able-bodied subjects. Stimulation also provides activation of afferent pathways that are timed with the activity of arm.

Results from the UEFT (Figure 1 and Table 2) and the DT (Figure 3 and Table 3) indicated that FET when applied in hemiplegic subjects who had some residual function shortly after CVA promoted functional recovery of the paretic arm and hand. Although the electrical stimulation was applied to distal muscles that controlled the opening grasping, holding, and releasing of the objects, the significant improvement was also noticed in elbow and shoulder joint movements.

The active exercise and electrical stimulation were applied during the period of the most prominent spontaneous recovery; thus, one could argue that the recovery was not caused by treatment. However, the differences between the control and FET groups suggest the opposite (Tables 2, 3, and 6). The FET promoted the recovery, and we suggest that actually FET during the spontaneous recovery was instrumental for extraordinary high gains in functioning.

The UEFT measured the subject's ability to use objects typical for daily activities (drinking, eating, writing, etc.). Able-bodied subjects could repeat selected functional tasks an average of 40 times during a $2 \mathrm{~min}$ interval. The most advanced group (FET HFG) succeeded to repeat the functional tasks an average 30 times at the end of the study, which is about 20 percent slower compared with able-bodied subjects. The subjects from control HFG were slower; they were able to repeat tasks up to about 15 times during a 2 min interval.

The hemiplegic subjects who had very limited functioning at the start of the study (LFG) had major problems in accomplishing any task at the start, and their recovery was much slower. The LFG subjects showed improvement, yet the gains in the UETF and DT were not as high as in the HFG. Possibly, a longer treatment is required for those hemiplegic subjects to achieve a larger gain. This conclusion follows that the trends lines have the largest slope during the 3 weeks of treatment (Table 6). 
Table 3.

Averaged normalized scores of Drawing Test of a one-way ANOVA between FET and control groups expressed in percent showing ability of subjects to coordinate shoulder and elbow joint measured at start and after 3, 6, 13, and 26 weeks of study for HFG and LFG groups. Each subject was asked to draw a square on a digitizing board with his or her paretic hand and arm. We obtained a score by dividing area subject drew with area of targeted square $\left(20 \mathrm{~cm} \times 20 \mathrm{~cm}=400 \mathrm{~cm}^{2}\right)$.

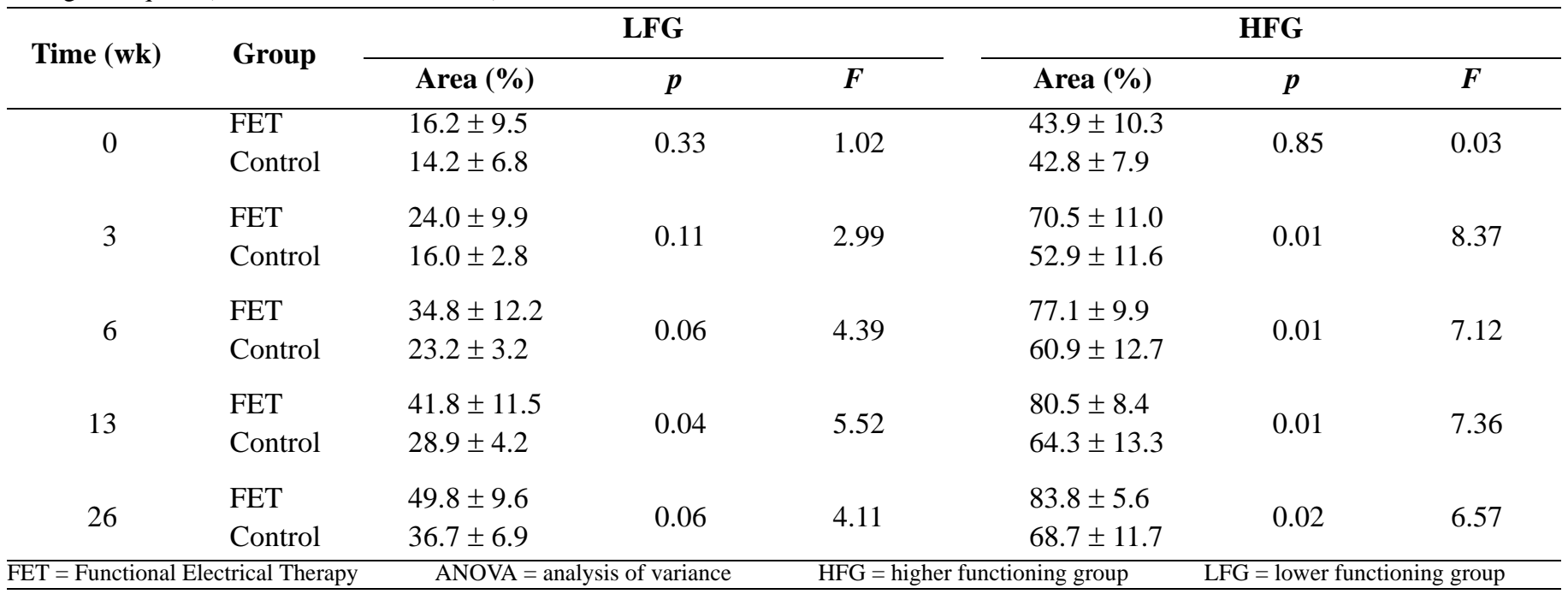

Table 4.

Averaged Ashworth grades for paretic arm measured at start of study (first row) and at end of study (second row). Grades were numerically adjusted to allow averaging; that is, value 0.25 was assigned instead of "+," and value 0.5 when grade was in between two integers (e.g., 2-3 was quantified as 2.5 ). Only statistically significant improvement was assessed in FET HFG.

\begin{tabular}{ccccc}
\hline $\begin{array}{c}\text { Time } \\
(\text { wk) }\end{array}$ & FET LFG & $\begin{array}{c}\text { Control } \\
\text { LFG }\end{array}$ & FET HFG & $\begin{array}{c}\text { Control } \\
\text { HFG }\end{array}$ \\
\hline 0 & $2.75 \pm 0.5$ & $2.5 \pm 0.75$ & $2.25 \pm 1$ & $2.5 \pm 1$ \\
26 & $2.5 \pm 0.75$ & $2.25 \pm 0.75$ & $1.25 \pm 0.5$ & $2.25 \pm 0.75$ \\
\hline $\boldsymbol{p}$ & $<0.29$ & $<0.59$ & $<0.05$ & $<0.27$ \\
$\boldsymbol{F}$ & 0.8 & 0.8 & 8.2 & 0.9
\end{tabular}

FET $=$ Functional Electrical Therapy

$\mathrm{HFG}=$ higher functioning group

$\mathrm{LFG}=$ lower functioning group

The trend lines in the UEFT and DT were obtained with the use of logarithmic fitting (Figures 1 and 3), since the improvements in functioning changed during the study (Table 6). The improvements were much larger during the treatment in the FET groups compared with control groups ( $p<0.01, F=7.8$ ). This finding indicated that FET sped up and promoted the recovery of functioning of the paretic arm and hand when compared with only exercise. During the period after the treatment, the trend lines slopes were comparable, yet at different levels (Table 6).
Table 5.

Averaged percent of maximum score of Amount Scale and How-Well Scale in Reduced Upper Extremity Motor Activity Log questionnaire that included 12 tasks.

\begin{tabular}{|c|c|c|c|c|}
\hline \multirow{2}{*}{$\begin{array}{l}\text { Time } \\
\text { (wk) }\end{array}$} & \multicolumn{4}{|c|}{$\%$ of Maximum Score-Amount Scale } \\
\hline & FET LFG & $\begin{array}{c}\text { Control } \\
\text { LFG }\end{array}$ & FET HFG & $\begin{array}{c}\text { Control } \\
\text { HFG }\end{array}$ \\
\hline 0 & 0 & 0 & $13.1 \pm 4.5$ & $12.6 \pm 6.5$ \\
\hline 26 & $16.7 \pm 8.3$ & $3.3 \pm 1.7$ & $59.7 \pm 12.5$ & $28.7 \pm 11.7$ \\
\hline$p$ & $<0.09$ & $<0.03$ & $<0.01$ & $<0.04$ \\
\hline $\boldsymbol{F}$ & 4.5 & 3.9 & 6.4 & 3.1 \\
\hline \multirow{2}{*}{$\begin{array}{l}\text { Time } \\
\text { (wk) }\end{array}$} & \multicolumn{4}{|c|}{ \% of Maximum Score-How-Well Scale } \\
\hline & FET LFG & $\begin{array}{c}\text { Control } \\
\text { LFG }\end{array}$ & FET HFG & $\begin{array}{c}\text { Control } \\
\text { HFG }\end{array}$ \\
\hline 0 & 0 & 0 & $16.5 \pm 5.5$ & $9.6 \pm 6.6$ \\
\hline 26 & $11.5 \pm 6.1$ & $2.3 \pm 1.2$ & $66.7 \pm 11.4$ & $32.5 \pm 10.6$ \\
\hline$p$ & $<0.09$ & $<0.03$ & $<0.01$ & $<0.04$ \\
\hline $\boldsymbol{F}$ & 3.2 & 4.1 & 7.9 & 3.2 \\
\hline
\end{tabular}

FET $=$ Functional Electrical Therapy

$\mathrm{HFG}=$ higher functioning group

$\mathrm{LFG}=$ lower functioning group

An important result was the measurable decrease of muscle tonus using the Ashworth scale grading of the key muscles of the paretic arm (Table 4). The key muscles tested were responsible for upper arm and forearm movements. The estimated difference based on the Ashworth 
Journal of Rehabilitation Research and Development Vol. 40, No. 5, 2003

Table 6.

Slopes of trend lines in Upper Extremity Function Test (UEFT) and Drawing Test (DT) for first 3 weeks (treatment period) and follow-up (remaining 23 weeks of study). Slopes were larger for FET groups compared to control groups during treatment, and overall gains were larger for FET groups compared with control groups across 26 weeks.

\begin{tabular}{|c|c|c|c|c|c|}
\hline \multirow{2}{*}{ Time (wk) } & \multirow{2}{*}{ Group } & \multicolumn{2}{|c|}{$\begin{array}{c}\text { UEFT Trend Line Slope } \\
\text { (No. of Repetitions in } 2 \mathrm{~min} / \mathrm{wk} \text { ) }\end{array}$} & \multicolumn{2}{|c|}{$\begin{array}{l}\text { DT Trend Line Slope } \\
\text { (\% Area/wk) }\end{array}$} \\
\hline & & LFG & HFG & LFG & HFG \\
\hline \multirow{2}{*}{$0-3$ (Treatment) } & FET & 0.65 & 4.6 & 2.5 & 9.0 \\
\hline & Control & 0.05 & 0.2 & 0.5 & 3.0 \\
\hline \multirow{2}{*}{ 3-26 (Follow-Up) } & FET & 0.15 & 0.5 & 1.1 & 0.5 \\
\hline & Control & 0.45 & 0.25 & 1.0 & 1.0 \\
\hline
\end{tabular}

scale between the FET and controls clearly shows that FET was more effective than only exercise $(p<0.01)$. The Ashworth scale grading depends on many subjective factors, and the scale is not sensitive enough to provide better insight into the changes of spasticity. Our results that show electrical stimulation helps in reduction of spasticity follow the findings from several previous studies $[16,30,31]$.

Results from the DT showed gains in elbow and shoulder functions. These changes in most hemiplegic subjects resulted in improved postural control.

The RUE/MAL results (Table 5) indicated that the hemiplegic subjects received FET quite well. FET promoted an increased usage of the paretic arm and hand. The HFG and LFG hemiplegic subjects reached higher satisfaction. The gains in LFG were not high enough to make the paretic arm and hand fully functional, especially if the paretic hand was not the dominant one (handedness).

The UEFT and DT SDs were larger toward the end of the 26 weeks, indicating that the differences among the subjects in both groups became substantial, although the groups matched at the start. Increased SDs show that the individual gains in some hemiplegic subjects were much larger or smaller compared with the averaged gains.

Finally, the gains in some subjects were extraordinarily high. This finding suggests that the improvement should be attributed to a combination of spontaneous recovery, FET, and activities that the subjects performed voluntarily as we hypothesized.

\section{REFERENCES}

1. Glanz M, Klawansky S, Stason W, Berkey C, Chalmers TC. Functional electrostimulation in post stroke rehabilita- tion: a meta-analysis of the randomised controlled trials. Arch Phys Med Rehabil 1996;77(6):549-53.

2. Chae J, Yu D. A critical review of neuromuscular electrical stimulation for treatment of motor dysfunction in hemiplegia. Assist Technol 2000;12(1):33-49.

3. Kroon JR, Lee JH van der, IJzerman MJ, Lankhorst GJ. Therapeutic electrical stimulation to improve motor control and functional abilities of the upper extremity after stroke: a systematic review. Clin Rehabil 2002;16(4):350-60.

4. Popovic DB, Popovic MB, Sinkjær T. Neurorehabilitation of upper extremities in humans with sensory-motor impairment. Neuromod 2002;5(1):54-67.

5. Sunderland A, Tinson DJ, Bradley EL, Fletcher D, Hewer RL, Wade DT. Enhanced physical therapy improves recovery of arm function after stroke-a randomised controlled trial. J Neurol Neurosurg Psychiatry 1992;55(7):530-35.

6. Wolf SL, Lecraw DE, Barton LA, Jann BB. Forced use of hemiplegic upper extremities to reverse the effect of learned nonuse pattern among chronic stroke and headinjured patients. Exp Neurol 1989;104(2):125-32.

7. Taub E, Uswatte G, Pidikiti R. Constraint-Induced Movement Therapy: a new family of techniques with broad application to physical rehabilitation-A clinical review. J Rehabil Res Dev 1999;36(3):237-51.

8. Kunkel A, Kopp B, Müller G, Villringer K, Villringer A, Taub E, Flor H. Constraint-induced movement therapy for motor recovery in chronic stroke patients. Arch Phys Med Rehabil 1999;80(6):624-28.

9. van der Lee JH, Wagenar RC, Lankhorst GJ, Vogelaar TW, Deville WL, Bouter LM. Forced use of the upper extremity in chronic stroke patients: results from a single blind randomised clinical trial. Stroke 1999;30:2369-75.

10. Volpe BT, Krebs HI, Hogan N, Edelstein L, Diels C, Aisen ML. A novel approach to stroke rehabilitation: robot-aided sensorimotor stimulation. Neurology 2000; 54(10):1938-44.

11. Kraft GH, Fitts SS, Hammond MC. Techniques to improve function of the arm and hand in chronic hemiplegia. Arch Phys Med Rehabil 1992;73(3):220-27. 
12. Francisco G, Chae J, Chawla H, Kirshblum S, Zorowitz R, Lewis G, Pang S. Electromyogram-triggered neuromuscular stimulation for improving the arm function of acute stroke survivors: a randomised pilot study. Arch Phys Med Rehabil 1998;79(5):570-75.

13. Chae J, Bethoux F, Bohine T, Dobos L, Davis T, Friedl A. Neuromuscular stimulation for upper extremity motor and functional recovery in acute hemiplegia. Stroke 1998; 29(5):975-79.

14. Cauraugh J, Light K, Kim S, Thigpen M, Behrman A. Chronic motor dysfunction after stroke: recovering wrist and finger extension by electromyography-triggered neuromuscular stimulation. Stroke 2000;31(6):1360-64.

15. Thorsen R, Spadone R, Ferrarin M. A pilot study of myoelectrically controlled FES of upper extremity: tracking test on SCI and stroke patients. IEEE Trans Rehabil Eng 2001;TRE-9(2):161-68.

16. Dimitrijevic MM, Stokic DS, Wawro AW, Wun CC. Modification of motor control of wrist extension by mesh-glove electrical afferent stimulation in stroke patients. Arch Phys Med Rehabil 1996;77(3):252-58.

17. Sonde L, Gip C, Fernaeus SE, Nilsson CG, Viitanen M. Stimulation with low frequency $(1.7 \mathrm{~Hz})$ transcutaneous electric nerve stimulation (low-tens) increases motor function of the post-stroke paretic arm. Scand J Rehabil Med 1998;30:295-99.

18. Sonde L, Kalimo H, Fernaeus SE, Viitanen M. Low TENS treatment on post-stroke paretic arm: a three-year followup. Clin Rehabil 2000;14:114-19.

19. Merlette R, Acimovic R, Grobelnik S, Cvilak G. Electrophysiological orthosis for the upper extremity in hemiplegia: feasibility study. Arch Phys Med Rehabil 1975;56(12): 507-13.

20. Popovic DB, Sinkjær T, Popovic MB, Stefanovic A, Pjanovic A, Schwirtlich L. Functional Electrical Therapy (FET) for improving the reaching and grasping in hemiplegics. Proceedings of the 6th IFESS Conference; Cleveland, Ohio. Cleveland: Case Western Reserve University; 2001. p. 108-10.

21. Popovic MB, Popovic DB, Sinkjær T, Stefanovic A, Schwirtlich L. Restitution of reaching and grasping pro- moted by Functional Electrical Therapy. Artif Organs 2002;26(3):271-75.

22. Popovic DB, Popovic MB, Sinkjær T. Neurorehabilitation of reach and grasp: Carry over effects induced by Functional Electrical Therapy. In: Gantchev N, editor. Basic motor control to functional recovery II. 2001. p. 413-23.

23. Bobath B. Adult hemiplegia: Evaluation and treatment. 3rd ed. London: Heinemann Medical Books Ltd.; 1990.

24. Caroll D. A quantitative test of upper extremity function. J Chronic Dis 1965;18:479-82.

25. Wijman CA, Stroh KC, Van Doren CL, Thrope GB, Peckham PH, Keith MW. Functional evaluation of quadriplegic patients using a hand neuroprosthesis. Arch Phys Med Rehabil 1990;71:1053-57.

26. Popovic DB, Popovic MB, Mladenovic S. Control of reaching: aligning of the hand velocity to the hand-target direction, vol. IV. Proceedings of the 41th Yugoslav Conference on ETRAN; Zlatibor, Yugoslavia. Belgrade: Yugoslav Committee for ETRAN; 1997. p. 241-44.

27. Eder C, Popovic MB, Chen AC, Popovic DB. A method for assessment of functional abilities in hemiplegic subjects: connectivism between ARM coordination and EMG. Proceedings of the World Conference EMBEC '02; 2002 Dec 4-8; Vienna, Austria. Verein Zur Veraustelung der EMBEC; 2002. p. 806-7.

28. Bohannon RW, Smith MB. Interrater reliability of a modified Ashworth scale of muscle spasticity. Phys Ther 1987; 67(2):206-7.

29. Blanton S, Wolf SL. An application of upper-extremity constraint-induced movement therapy in a patient with subacute stroke. Phys Ther 1999;79(9):847-53.

30. Dewald JP, Given JD, Rymer WZ. Long-lasting reductions of spasticity induced by skin electrical stimulation. IEEE Trans Rehabil Eng 1996;4(4)231-42.

31. Daly JJ, Marsolais EB, Mendell LM, Rymer WZ, Stefanovska A, Wolpaw JR, Kantor C. Therapeutic neural effects of electrical stimulation. IEEE Trans Rehabil Eng 1996;4(4)218-30.

Submitted for publication March 14, 2002. Accepted in revised form March 10, 2003. 